SCIENTIFIC LETTER

\title{
Long term follow up of patients with repaired aortic coarctations
}

\author{
J P de Bono, L J Freeman
}

Heart 2005;91:537-538. doi: 10.1136/hrt.2004.037663

A ortic coarctation was one of the first types of congenital heart diseases to be treated successfully with surgery. Despite initial optimism, surgical relief of aortic narrowing does not cure the underlying vascular problem. Patients with previously repaired coarctation have a significantly reduced life expectancy and continue to be at risk of major complications throughout life including hypertension, aortic aneurysms, premature coronary artery disease, aortic valve problems, and cerebral aneurysms. ${ }^{12}$ In addition female patients are at particular risk during pregnancy and childbirth. ${ }^{3}$ All patients with previously repaired aortic coarctation require careful life long follow up. ${ }^{2}{ }^{4}$ Without follow up they may present too late with untreated hypertension or with catastrophic complications such as aortic dissection. Although aortic coarctation may be dismissed as a rare condition of little general interest, most primary care groups in the UK will have at least one patient with aortic coarctation.

We carried out a notes based study of patients with known aortic coarctation to assess the adequacy of long term follow up.

\section{PATIENTS AND METHODS}

We identified 53 patients with previously repaired aortic coarctation attending either general cardiology clinics or the specialist adult congenital heart disease clinics at the Norfolk and Norwich University Hospital, a large district general hospital with no cardiac surgery facilities and only visiting paediatric cardiologists. Notes were obtained for all patients including, where possible, notes from other hospitals where patients had previously been treated.

\section{RESULTS}

Four patients with a previous repair of aortic coarctation were still under the care of general adult cardiologists. The remainder were seen in the specialist adult congenital heart disease clinic. Over a third of those referred to the adult congenital heart disease clinic with a previous coarctation repair $(34 \%, 17 / 49)$ were not under any active follow up at the time of referral, mainly by primary care physicians. The remaining 32 patients had been referred for further specialised follow up from either the paediatric (14 patients) or adult cardiologists (17 patients) or other adult congenital heart disease services (one patient).

In total $42 \%(22 / 53)$ of patients with a previous coarctation repair had at some point been lost to any cardiology follow up for a period of at least two years. Thirty per cent (16/53) had actually been discharged from a clinic by non-specialised cardiologists, including four patients within the last five years. Of those patients not under regular follow up, nine had been referred to the adult congenital heart disease clinic with presumed complications of their previous coarctation repair, while two were pregnant.

\section{DISCUSSION}

Patients with repaired coarctations have a high risk of further complications and a significantly reduced life expectancy. Long term specialist follow up of patients with repaired aortic coarctation allows early detection and treatment of complications including hypertension, recoarctation, repair site aneurysms, and aortic root problems, often before they are symptomatic, and allows catastrophic events to be prevented. ${ }^{245}$ Ongoing specialist management of female patients also allows careful planning and monitoring of pregnancy from the pre-conception stage to delivery.

This study shows that a significant number of patients with previous coarctation repair are not under active follow up and only return to specialist care fortuitously or when problems arise. There are likely to be many more such patients, unrecognised in the community. Patients with repaired coarctation are still being discharged from adult cardiology clinics. Such patients may only return to specialised care with catastrophic events or when symptomatic with previously silent complications. ${ }^{5}$ Unless the supervising cardiologist appreciates the complications likely to occur, then best patient care will occur when they are followed up by those with special training and interest in congenital heart disease in the adult. Primary care physicians should actively seek out patients with previously repaired aortic coarctation on their lists. Hospital doctors should be aware that previous aortic coarctation repair is not simply past medical history in patients presenting with medical problems. Both should ensure that these patients are seen and followed up in a consultant led specialist service and certainly not by juniors in training. Cardiologists who do not specialise in congenital heart disease should either refer their patients on to other cardiologists with an interest in the long term management of aortic coarctation or, if this is not feasible, they should work with them to devise protocol driven care pathways paying particular attention to monitoring of repair sites and aortic valve disease, pre- and peri-pregnancy counselling and care, and aggressive monitoring and control of blood pressure and other risk factors for ischaemic heart disease. Paediatric cardiologists need to work with their specialised adult colleagues to ensure a seamless transition of care to the most appropriate ongoing care for each individual patient.

\section{Authors' affiliations}

J P de Bono, L J Freeman, GUCH unit, Norfolk and Norwich University Hospital, Norwich, Norfolk, UK

Correspondence to: Dr Leisa J Freeman, GUCH unit, Norfolk and Norwich University Hospital, Norwich, Norfolk NR4 7UY, UK; leisa.freeman@nnuh.nhs.uk

Accepted 26 July 2004 


\section{REFERENCES}

1 Cohen M, Fuster V, Steele PM, et al. Coarctation of the aorta: long term follow up and prediction of outcome after surgical correction. Circulation 1989:80:840-54

2 Roos-Hesselink JW, Scholzel BE, Heijdra RJ, et al. Aortic valve and aortic arch pathology after coarctation repair. Heart 2003;89:1074-7.
3 Celermajer DS, Greaves K. Survivors of coarctation repair: fixed but not cured. Heart 2002;88:113-4.

4 Venning S, Freeman $\sqcup$, Stanley K. Two cases of pregnancy with coarctation of the aorta. J R Soc Med 2003;96:234-6.

5 Menon D, Burdge T. Haemoptysis as a late presentation of aneurysm leakage after aortic coarctation repair: the case for vigilant lifelong radiology surveillance? Emerg Med J 2003;20:102-3.

\section{IMAGES IN CARDIOLOGY}

\section{Triphasic left ventricular filling in hypertrophic cardiomyopathy}

Tring images show a triphasic pattern of left ventricula (LV) filling in a symptomatic 15 year old patient with non-obstructive hypertrophic cardiomyopathy. This pattern of LV filling is unusual. The sequence clearly demonstrated three separated filling waves. The underlying mechanism seems to be a mixed pattern of restrictive filling and delayed impaired relaxation. The tissue Doppler image in this case is compatible with the recognised diastolic asynchronisation found in hypertrophic cardiomyopathy.
The third annulus longitudinal movement may be caused by an extra myocardial relaxation, with suction capacity.
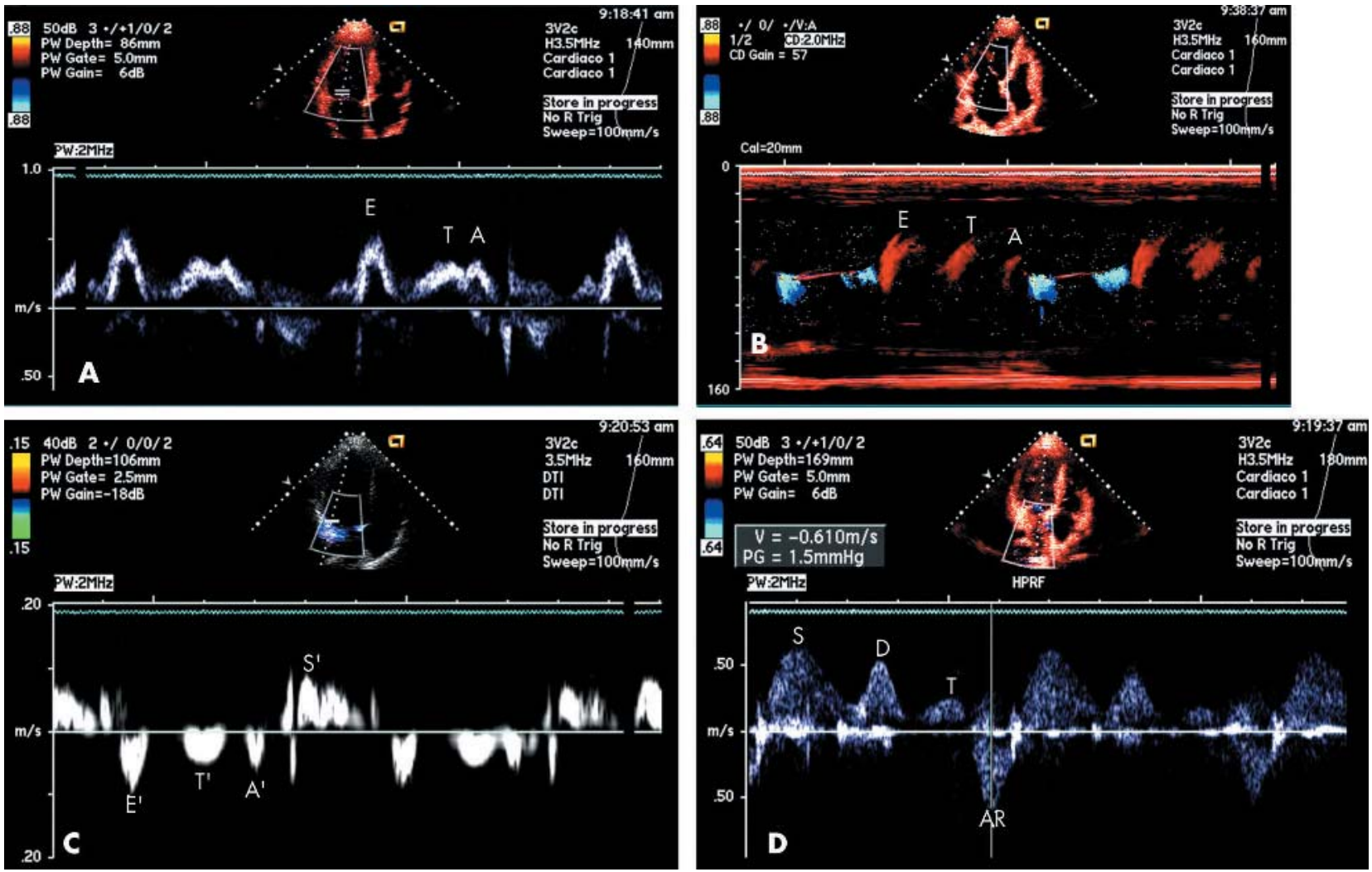

Panel A: mitral Doppler inflow. Between early $(E)$ and atrial $(A)$ waves, there is a third wave $(T)$ indicative of a mid diastolic filling. Panel B: M colour flow mapping. The mid diastolic filling wave (T) is also evident, and well separated from E and A waves. Panel C: pulsed tissue Doppler of the lateral mitral annulus. During left ventricular systole, the longitudinal movement is toward the apex $\left(\mathrm{S}^{\prime}\right)$. In diastole, the early velocity $\left(\mathrm{E}^{\prime}\right)$ is low $(<10 \mathrm{~cm} / \mathrm{s})$ reflecting impaired relaxation. Before the late atrial velocity $\left(A^{\prime}\right)$, there is a clear and discriminated mid diastolic annular movement $\left(T^{\prime}\right)$. Panel $D$ : pulmonary venous Doppler flow. The systolic (S) and early diastolic (D) waves are well delineated. The atrial reverse wave (AR) has a high peak velocity $(>50 \mathrm{~cm} / \mathrm{s})$. Between the two diastolic waves (D and AR), there is a third (T) wave. 